\title{
Avaliação nutricional de crianças com doença renal crônica
}

\author{
Nutritional status of children with chronic renal failure
}

Ana Paula Brecheret ${ }^{1}$, Ulysses Fagundes ${ }^{1}$, Marise Lazaretti Castro ${ }^{2}$, Maria Cristina Andrade ${ }^{3}$, João Tomás de A. Carvalhaes ${ }^{4}$

\section{RESUMO}

Objetivo: Desnutrição constitui uma frequente complicação em crianças portadoras de doença renal, sendo a baixa estatura o principal sinal clínico. O objetivo deste estudo é analisar o estado nutricional de crianças portadoras de doença renal por meio da antropometria.

Métodos: Estudo transversal que avaliou 21 (43\%) meninos e 28 (57\%) meninas, com idades entre 5,3 e 19,5 anos. As crianças foram divididas em três grupos, de acordo com o clearance $(\mathrm{mL} / \mathrm{min} / 1,73 \mathrm{~m} 2)$ : Grupo 1, clearance >37 ( $n=19)$; Grupo 2, entre 15 e $37(n=9)$ e Grupo 3 com clearance $<15(n=21)$. A partir do peso e da estatura, calcularam-se os seguintes indicadores: Peso/Idade (P/I), Estatura/Idade (E/I) e índice de massa corpórea (IMC) e obtiveram-se os escores $Z$ respectivos. Escores $Z$ menores que -2 foram considerados desnutrição. Os grupos foram comparados por ANOVA.

Resultados: Não se evidenciaram diferenças entre os grupos no que diz respeito aos dados antropométricos. 19 pacientes $(38,8 \%)$ apresentaram baixa estatura e $22(44,8 \%)$, baixo peso. Também não se observaram diferenças entre os grupos quando comparados pelos valores dos indicadores $\mathrm{P} / \mathrm{I}, \mathrm{E} / \mathrm{I}$ e IMC. Os valores dos escores $\mathrm{Z}$ para os indicado-

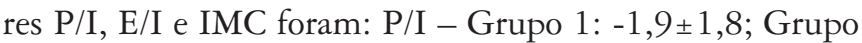
2: $-2,6 \pm 3,1 ;$ Grupo 3: $-2,5 \pm 1,4(p=0,47)$; E/I - Grupo 1: $-1,5 \pm 1,2$; Grupo 2: $-2,3 \pm 1,8$; Grupo 3: $-2,1 \pm 1,1 \quad(p=0,18)$ e IMC - Grupo 1: $-1,2 \pm 1,4$; Grupo 2: $-1,7 \pm 3,9$; Grupo 3: $-1,6 \pm 1,3(p=0,82)$.

Conclusões: A amostra estudada apresentou alta prevalência de desnutrição. Mesmo considerando-se o estágio da doença, não se encontraram diferenças significativas entre os grupos.

Instituição: Universidade Federal de São Paulo (Unifesp), São Paulo, SP, Brasil

${ }^{1}$ Mestres em Ciências pelo Departamento de Pediatria da Escola Paulista de Medicina da Universidade Federal de São Paulo (Unifesp-EPM), São Paulo, SP, Brasil

${ }^{2}$ Livre-docente pelo Departamento de Medicina da Unifesp-EPM, São Paulo, SP, Brasil

${ }^{3}$ Doutora em Ciências pelo Departamento de Pediatria da Unifesp-EPM, São Paulo, SP, Brasil

${ }^{4}$ Professor Adjunto; Chefe do Setor de Nefrologia Pediátrica do Departamento de Pediatria da Unifesp-EPM. São Paulo, SP, Brasil
Palavras-chave: insuficiência renal crônica; avaliação nutricional; criança; adolescente.

\section{ABSTRACT}

Objective: Malnutrition is a frequent complication among children with renal diseases. Short stature is the main clinical sign. The aim of this study is to analyze the nutritional status of children with renal disease using anthropometry.

Methods: This cross sectional study enrolled 21 (43\%) boys and $28(57 \%)$ girls with age ranging from 5.3 to 19.5 years. They were divided in three groups based on their creatinine clearance $\left(\mathrm{mL} / \mathrm{min} / 1.73 \mathrm{~m}^{2}\right)$ : Group $1,>37$ $(\mathrm{n}=19)$; Group 2, between 15 and $37(\mathrm{n}=9)$ and Group 3, $<15(\mathrm{n}=21)$. Weight and height were obtained in order to calculate the following indexes: Weight/age (W/A), height/ age (H/A) and body mass index (BMI); then, $\mathrm{Z}$ scores were obtained. Malnutrition was defined as Z scores below -2 . ANOVA test was used to compare groups.

Results: There were no differences among the groups for anthropometric data. 19 patients (38.8\%) presented short-stature and $22(44.8 \%)$ low-weight. Z scores were similar among groups relative to $\mathrm{W} / \mathrm{A}, \mathrm{H} / \mathrm{A}$ and $\mathrm{BMI}$ values. W/A Z score values were: Group 1: $-1.9 \pm 1.8$; Group 2: $-2.6 \pm 3.1$ and Group 3: $-2.5 \pm 1.4(p=0.47)$. H/A Z scores values were: Group 1: $-1.5 \pm 1.2$; Group 2: $-2.3 \pm 1.8$ and Group 3: $-2.1 \pm 1.1(p=0.18)$. The calculated BMI Z scores were: Group 1: $-1.2 \pm 1.4$; Group 2: $-1.7 \pm 3.9$ and Group 3: $-1.6 \pm 1.3(p=0.82) .19$ children presented short stature and 22 presented low weight. There were no differences between the studied groups.

Endereço para correspondência:

Ana Paula Brecheret

Rua Florália, 79 - Vila Madalena

CEP 05451-130 - São Paulo/SP

Email: paulabrecheret@uol.com.br

Recebido em: 19/5/08

Aprovado em: 2/11/08 
Conclusions: The sample presented high prevalence of malnutrition. Even considering the disease stage, there were no nutritional differences between the studied groups.

Key-words: renal insufficiency, chronic; nutritional assessment; child; adolescent.

\section{Introdução}

A doença renal crônica (DRC) é uma síndrome clínica causada pela perda progressiva e irreversível das funções renais. Sua etiologia varia com a idade. Nos recém-nascidos e lactentes as malformações do trato urinário são as predominantes, enquanto nos escolares e adolescentes prevalecem as glomerulopatias ${ }^{(1-4)}$.

O quadro clínico da DRC terminal (também chamado de uremia) só começa a se desenvolver com a perda funcional de mais de $75 \%$ dos glomérulos. A uremia é caracterizada por um conjunto de manifestações clínicas e bioquímicas dependentes de alterações da função renal. Na DRC, a evolução para estágios terminais e, necessariamente, para terapia substitutiva renal, transplante ou diálise, é inexorável ${ }^{(1-3)}$.

A insuficiência renal causa um estado de desajuste metabólico proporcional à queda da função renal, com alterações enzimáticas responsáveis pela diminuição da síntese protéica, por alterações na tolerância aos carboidratos e na produção de energia celular, além de alterações no balanço de água, eletrólitos, no equilíbrio ácido-básico, no metabolismo de lipídeos e na produção de energia celular ${ }^{(1-3)}$.

Independentemente da etiologia, todas as crianças afetadas precisam de cuidados nutricionais específicos. A terapia nutricional exerce importante papel na redução e até na estabilização do ritmo de progressão da insuficiência renal. Quando a filtração glomerular e o balanço glomérulo-tubular já estão gravemente comprometidos, limitações e alterações na dieta podem ajudar a prevenir distúrbios metabólicos ${ }^{(1,3,5,6)}$. O estado nutricional é ainda um importante fator prognóstico nos portadores de DRC que, quando desnutridos, têm risco de morbidade e a mortalidade aumentado, além de pior qualidade de vida se comparados a seus pares eutróficos ${ }^{(7-10)}$.

Nas crianças com DRC, a desnutrição energético-proteica é um achado frequente, contribuindo para as elevadas taxas de baixa estatura nesta população ${ }^{(3,5,11)}$. Estudo realizado por Pereira e Carvalhaes, em ambulatório de nefrologia pediátrica em 1994, encontrou algum grau de desnutrição em 100\% dos pacientes acompanhados ${ }^{(12)}$. A baixa estatura é uma importante manifestação da desnutrição na DRC e ocorre em cerca de $50 \%$ das crianças que iniciam o tratamento dialítico. A gravidade do retardo de crescimento está relacionada à idade de início da insuficiência renal, ao clearance de creatinina e ao grau de desnutrição desses pacientes ${ }^{(5)}$.

A necessidade do suporte nutricional como parte do tratamento da DRC já está bem estabelecida na literatura. Entre os métodos utilizados para a avaliação nutricional, a antropometria, com as medidas de peso e estatura, tem sido o método mais frequentemente usado no acompanhamento desses pacientes.

Este trabalho tem como objetivo avaliar o estado nutricional de crianças e adolescentes portadores de DRC por meio da antropometria e comparar o estado nutricional dos pacientes com DRC em tratamento conservador com clearance de creatinina $\geq 37 \mathrm{~mL} / \mathrm{min} / 1,73 \mathrm{~m}^{2}$ às crianças e adolescentes com DRC em tratamento conservador com clearance de creatinina menor do que $<37 \mathrm{~mL} / \mathrm{min} / 1,73 \mathrm{~m}^{2}$ e àquelas em diálise.

\section{Métodos}

Após aprovação do Comitê de Ética em pesquisa da Unifesp-EPM, 49 crianças em acompanhamento no ambulatório de nefrologia pediátrica do Hospital São Paulo da UnifespEPM foram estudadas transversalmente, com idade média de $11,8 \pm 3,8$ anos, mediana de 11,7 anos (variação: 5,3 a 19,5 anos), sendo $21(42,8 \%)$ meninas e $28(57,1 \%)$ meninos. Os dados foram colhidos de novembro de 2003 a setembro de 2004. A amostra foi selecionada, por conveniência, no ambulatório de nefrologia pediátrica, respeitando-se os critérios de inclusão e exclusão. Foram incluídos todos os pacientes do ambulatório de doença renal crônica do serviço de nefrologia pediátrica entre quatro e 21 anos de idade e apenas quatro deles se encaixavam nos critérios de exclusão.

Como critério de inclusão, considerou-se pacientes portadores de DRC (isto é, ter clearance de creatinina $<75 \mathrm{~mL} / \mathrm{min} / 1,73 \mathrm{~m}^{2}$ ou estar em programa de diálise crônica há mais de dois meses) e idade superior a quatro anos e $\leq 21$ anos. Usou-se como ponte de corte inferior a idade de quatro anos para evitar incluir, no mesmo grupo, lactentes e adolescentes. Excluíram-se pacientes com deformidades físicas que impossibilitassem a medida da estatura e aqueles com internação prévia nos últimos 30 dias.

Para verificar a presença de diferenças de estado nutricional e de composição corporal das crianças em tratamento conservador e dialítico, os pacientes foram distribuídos em três grupos, chamados: Conservador 1, que incluiu pacientes em 
tratamento conservador, com clearance $\geq 37 \mathrm{~mL} / \mathrm{min} / 1,73 \mathrm{~m}^{2}$; Conservador 2 , que incluiu pacientes em tratamento conservador com clearance $<37 \mathrm{~mL} / \mathrm{min} / 1,73 \mathrm{~m}^{2}$; e Diálise, que incluiu pacientes em diálise (clearance de creatinina $<15 \mathrm{~mL} / \mathrm{min} / 1,73 \mathrm{~m}^{2}$ ). Na divisão realizada para analisar os pacientes em tratamento conservador, procurou-se separar os mais graves dos menos graves, pois tais pacientes em tratamento conservador apresentam características clínicas heterogêneas, com agravamento das alterações típicas da doença principalmente naqueles com clearance de creatinina $<35-40 \mathrm{~mL} / \mathrm{min} / 1,73 \mathrm{~m}^{2(1,2,13)}$. Foram consideradas, em programa de diálise crônica, as crianças portadoras de DRC em diálise peritoneal ou em hemodiálise há mais de dois meses.

Após leitura e assinatura do termo de consentimento livre e esclarecido pelos pais ou responsáveis, realizou-se exame físico com verificação da presença ou não de edema clínico, medida de peso e estatura e avaliação do estágio puberal. Nas crianças em hemodiálise, a coleta de dados foi realizada até duas horas após o término da sessão de hemodiálise na segunda sessão da semana (quarta ou quinta-feira). Nas crianças em tratamento conservador e em diálise peritoneal, a coleta foi feita com data marcada, estando as crianças em diálise peritoneal com a cavidade peritoneal vazia.

Para o cálculo do clearance de creatinina foi utilizada a fórmula de Schwartz ${ }^{(14)}$, aplicando a estatura medida no momento da realização do exame e a creatinina dosada na última consulta de rotina do ambulatório.

Todas as medidas foram realizadas por um único pesquisador e a coleta de dados foi realizada de novembro de 2003 a setembro de 2004. Para aferição de peso, utilizou-se balança tipo plataforma da marca Filizola ${ }^{\circledR}$, com capacidade máxima de $150 \mathrm{~kg}$, mínima de $2,5 \mathrm{~kg}$ e precisão de $100 \mathrm{~g}$. As crianças foram pesadas, usando apenas roupas íntimas, por três vezes consecutivas ou até que se obtivessem dois valores com diferença menor ou igual a $100 \mathrm{~g}$. A estatura foi medida em antropômetro de madeira fixo de parede, com extensão de $200 \mathrm{~cm}$, graduação em centímetros e precisão de $1 \mathrm{~mm}$. As medidas consideradas válidas foram aquelas que coincidiam duas vezes ${ }^{(15)}$.

Para avaliar o estado nutricional das crianças estudadas, foram utilizadas as seguintes variáveis: peso, estatura, gênero e idade. Os indicadores do estado nutricional estudados foram: peso para a idade $(\mathrm{P} / \mathrm{I})$, estatura para idade $(\mathrm{E} / \mathrm{I})$ e índice de massa corpórea para a idade (IMC/I). Foram consideradas com baixo peso ou baixa estatura as crianças com escore $Z$ menor do que -2 de cada indicador e obesas, aquelas com escore $\mathrm{Z}$ acima de 2 , conforme os critérios da $\mathrm{OMS}^{(16)}$. Os valores obtidos foram comparados ao padrão do NCHS-2000 ${ }^{(16)}$, utilizando-se o programa Nutrition do software Epi-Info 2002 do Centers for Disease Control (CDC).

Os dados foram analisados em relação ao gênero dos pacientes e em relação aos grupos de paciente (Conservador 1 , Conservador 2 e Diálise). Os grupos não foram segmentados entre os gêneros, pois resultariam em poucos pacientes, não permitindo a comparação entre eles. Para comparar os grupos de paciente, empregou-se a Análise de Variância (ANOVA) com os softwares Epi-Info 2002 e SPSS 11.0.

\section{Resultados}

Os valores da idade, do clearance de creatinina e do tempo de diálise, segundo o tratamento, encontram-se na Tabela 1. A etiologia da DRC dos pacientes estudados é apresentada na Tabela 2. A distribuição das crianças e adolescentes estudados de acordo com o tratamento e o gênero encontra-se na Tabela 3 .

Os valores médios dos escores $\mathrm{Z}$ das relações $\mathrm{P} / \mathrm{I}, \mathrm{E} / \mathrm{I}$, IMC/I segundo o tratamento estão dispostos na Tabela 4, não havendo diferenças nutricionais entre os grupos analisados. A baixa estatura (escore $Z<-2$ ) foi diagnosticada em 19 $(38,8 \%)$ crianças, enquanto o baixo peso foi encontrado em 22

Tabela 1 - Valores da idade, do clearance de creatinina e do tempo de diálise dos pacientes segundo o tratamento, expressos em média \pm desvio padrão e em mediana (mínimo; máximo)

\begin{tabular}{|c|c|c|c|}
\hline Tratamento & $\begin{array}{l}\text { Idade } \\
\text { (anos) }\end{array}$ & $\begin{array}{l}\text { Clearance creatinina } \\
\left(\mathrm{mL} / \mathrm{min} / 1,73 \mathrm{~m}^{2}\right)\end{array}$ & $\begin{array}{c}\text { Tempo de diálise } \\
\text { (meses) }\end{array}$ \\
\hline \multirow[t]{2}{*}{ Conservador 1} & $13,0 \pm 4,0$ & $52,6 \pm 12,7$ & - \\
\hline & $13,8(5,3 ; 19,5)$ & $47,7(37,1 ; 69,9)$ & \\
\hline \multirow[t]{2}{*}{ Conservador 2} & $10,3 \pm 3,6$ & $25,4 \pm 4,8$ & - \\
\hline & $8,8(6,2 ; 15,7)$ & $24,4(19,0 ; 33,3)$ & \\
\hline Diálise & $11,3 \pm 3,6$ & - & $19,8 \pm 20,9$ \\
\hline
\end{tabular}

Tratamento versus idade; ANOVA: $p=0,181$ 
(44,8\%). O percentual de crianças com escore $Z<-2$ desvios padrão para os índices antropométricos P/I, E/I e IMC/I está disposto na Tabela 5. Nenhuma criança teve diagnóstico de obesidade, nem escore $Z>2$ desvios padrão para $\mathrm{E} / \mathrm{I}$ ou $\mathrm{P} / \mathrm{I}$. A Figura 1 apresenta os escores $Z$ dos pacientes estudados para os indicadores P/I, E/I e IMC/I.

O estágio puberal das crianças estudadas foi analisado através do teste qui-quadrado e não foi encontrada diferença

Tabela 2 - Distribuição dos pacientes estudados segundo a etiologia da DRC

\begin{tabular}{lrr}
\hline Etiologia & $\mathbf{n}$ & $\%$ \\
\hline Uropatia obstrutiva/nefropatia de refluxo & 17 & 34,7 \\
Glomerulopatias & 13 & 26,5 \\
Indeterminada & 7 & 14,3 \\
Tubulopatia & 5 & 10,2 \\
Displasia/aplasia renal & 2 & 4,1 \\
Síndrome hemolitico-urêmica & 2 & 4,1 \\
Tumor renal & 1 & 2,0 \\
Litíase renal & 1 & 2,0 \\
Lupus eritematoso sistêmico & 1 & 2,0 \\
\hline Total & 49 & 100,0 \\
\hline
\end{tabular}

Tabela 3 - Distribuição dos pacientes estudados segundo tratamento e gênero

\begin{tabular}{|c|c|c|c|c|c|c|}
\hline \multirow[t]{2}{*}{ Tratamento } & \multicolumn{2}{|c|}{ Masculino } & \multicolumn{2}{|c|}{ Feminino } & \multicolumn{2}{|c|}{ Total } \\
\hline & $\mathrm{n}$ & $\%$ & $\mathrm{n}$ & $\%$ & $\mathbf{n}$ & $\%$ \\
\hline Con & 12 & 24,5 & 7 & 14,3 & 19 & 38,8 \\
\hline Conserv & 7 & 14,3 & 2 & 4,0 & 9 & 18,4 \\
\hline Diálise & 9 & 18,4 & 12 & 24,5 & 21 & 42,8 \\
\hline Total & 28 & 57,2 & 21 & 42,8 & 49 & 100,0 \\
\hline
\end{tabular}

Qui-quadrado: $p=0,166$ estatística na comparação entre os gêneros $(p=0,148)$ ou entre tratamentos $(p=0,27)$.

\section{Discussão}

A desnutrição tem sido um grande desafio no tratamento da insuficiência renal em crianças, pois é responsável pelo aumento da mortalidade e morbidade, além de levar à baixa estatura, como visto na amostra estudada ${ }^{(7,9,10)}$. A antropometria é o método mais utilizado para o acompanhamento nutricional desses pacientes, com a vantagem de ser de fácil execução e de baixo custo ${ }^{(17,18)}$. Nos renais crônicos, o método antropométrico é dificultado pela presença de edema, principalmente nos pacientes em diálise, e por isso sua realização exige cuidados com o exame clínico e com o momento do exame, devendo ser feita de preferência logo após uma sessão de diálise efetiva.

Os estudos realizados em crianças com DRC geralmente são limitados pelo número de pacientes, pois se trata de

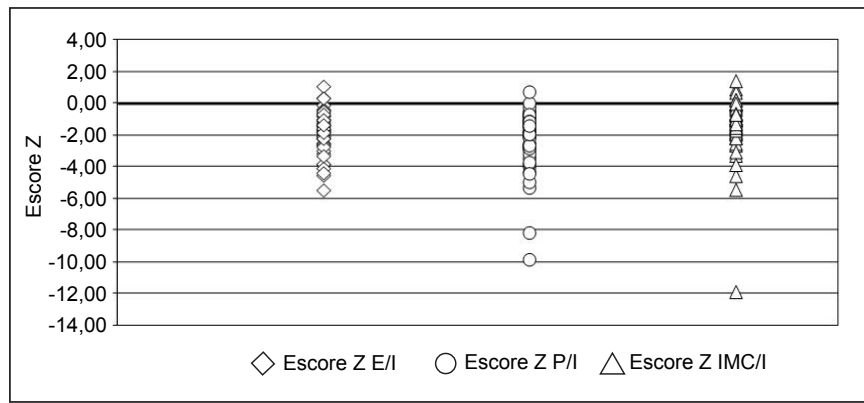

Figura 1 - Escores Z dos pacientes estudados para os indicadores peso/idade, estatura/idade e IMC/idade.

Tabela 4 - Valores dos escores Z das relações peso para idade (P/I), estatura para idade (E/I) e IMC para idade (IMC/I) segundo o tratamento, expressos em média \pm desvio padrão e em mediana (mínimo; máximo)

\begin{tabular}{lcccc}
\hline Relações & Conservador $\mathbf{1}$ & Conservador $\mathbf{2}$ & Diálise & ANOVA \\
\hline $\mathrm{P} / \mathrm{I}$ & $-1,9 \pm 1,8$ & $-2,6 \pm 3,1$ & $-2,5 \pm 1,4$ & 0,47 \\
$\mathrm{E} / \mathrm{I}$ & $-1,2(-8,2 ; 0,0)$ & $-1,4(-9,9 ;-0,5)$ & $-2,2(-5,0 ; 0,7)$ & \\
& $-1,5 \pm 1,2$ & $-2,3 \pm 1,8$ & $-2,1 \pm 1,1$ & 0,18 \\
$\mathrm{IMC} / \mathrm{I}$ & $-1,5(-4,6 ; 1,0)$ & $-2,6(-5,5 ; 0,3)$ & $-1,8(-4,4 ;-0,6)$ & \\
& $-1,2 \pm 1,4$ & $-1,7 \pm 3,9$ & $-1,6 \pm 1,3$ & 0,82 \\
\hline
\end{tabular}

Tabela 5 - Percentual de pacientes que apresentaram escores Z menores que -2 desvios-padrão, para os índices antropométricos $\mathrm{P} / \mathrm{I}, \mathrm{E} / \mathrm{I}$ e IMC/I, segundo tratamento

\begin{tabular}{lccccccccc}
\hline & \multicolumn{2}{c}{ Conservador $\mathbf{1}$} & \multicolumn{2}{c}{ Conservador $\mathbf{2}$} & \multicolumn{2}{c}{ Diálise } & \multicolumn{2}{c}{ Total } & Qui-quadrado \\
Índice & $\mathbf{n}$ & $\mathbf{\%}$ & $\mathbf{n}$ & $\mathbf{\%}$ & $\mathbf{n}$ & $\mathbf{\%}$ & $\mathbf{n}$ & $\mathbf{\%}$ & \\
\hline P/I & 6 & 31,6 & 3 & 33,3 & 13 & 61,9 & 22 & 44,8 & 0,12 \\
E/I & 6 & 31,6 & 5 & 55,6 & 8 & 38,1 & 19 & 38,8 & 0,48 \\
IMC/I & 3 & 15,8 & 1 & 11,1 & 6 & 28,6 & 10 & 20,2 & 0,45 \\
\hline
\end{tabular}


uma doença rara, com taxa de incidência de cerca de dez pacientes por milhão de pessoas menores de $20 \operatorname{anos}^{(19,20)}$. No presente trabalho, a casuística ( 49 crianças) constituiu-se de praticamente todas as crianças acompanhadas no ambulatório de doença renal crônica que se encaixavam nos critérios de inclusão, número este que pode ser comparado às casuísticas dos trabalhos apresentados na literatura ${ }^{(22,23)}$.

Para a análise dos dados, os pacientes foram distribuídos em três grupos conforme sugerido por Kopple et al. Apesar do último analisar adultos, foi o único encontrado da literatura que estudou esses parâmetros nutricionais e encontrou diferença a partir dos mesmos pontos de corte escolhidos no presente trabalho ${ }^{(13)}$. Não foi possível realizar a análise em crianças em hemodiálise e diálise peritoneal, pois o número de pacientes por grupo seria muito pequeno. Por esse mesmo motivo, optou-se por dividir a casuística das crianças em tratamento conservador em dois grupos, buscando separar aquelas em estágio mais ou menos avançado da doença, com menor ou maior clearance de creatinina.

Segundo estatística oficial, 8,4\% das crianças brasileiras apresentam baixo peso ${ }^{(21)}$. Na amostra estudada, a prevalência de baixo peso foi $44 \%$. Entre as crianças estudadas, 38,8\% apresentaram baixa estatura, enquanto na população brasileira a porcentagem é de $17,9 \%^{(21)}$. Esses resultados eram esperados, pois vão ao encontro dos dados da literatura, realçando-se o fato de que os efeitos da doença no crescimento predominam em relação à estatura, quando comparada ao peso $^{(22,23)}$. Ao analisar os valores do peso e da estatura respectivamente das crianças estudadas, verificou-se que $98 \%$ e $94 \%$ apresentaram valores menores que o percentil 50 da população de referência, pareada por idade e sexo para o peso para a estatura.

É importante salientar que os dados mostraram grande variabilidade, como indicam os valores de desvios padrão obtidos. Essa amplitude, entretanto, está concentrada nos valores negativos dos escores $Z$ obtidos. Isso pode ser observado nos valores de escore $Z$ para peso que, por exemplo, chegam a atingir -8,2 no Grupo Conservador 1, assim como o escore Z para IMC no Grupo Conservador 2 chega a -11,9.

Os resultados deste estudo mostraram não haver diferença entre os grupos estudados, indicando que, independentemente do clearance de creatinina ou de tratamento dialítico, os pacientes são desnutridos. Isso sugere que os pacientes já apresentavam desnutrição ao diagnóstico da doença renal crônica. Esses dados contrastam com os da literatura. Em trabalho feito com pacientes adultos, observou-se pior es- tado nutricional em pacientes com clearance de creatinina abaixo de $37 \mathrm{~mL} / \mathrm{min} / 1,73 \mathrm{~m}^{2(13)}$ e, em pesquisa realizada com crianças, constatou-se piora nos parâmetros antropométricos à medida que diminuía o clearance ${ }^{(24)}$. Uma característica dos pacientes que participaram do presente estudo é o diagnóstico tardio de insuficiência renal. Na casuística utilizada, 14,3\% têm etiologia indeterminada, indicando que quando chegaram ao serviço já estavam em DRC tardia. Outros 30,6\% eram portadores de uropatia congênita, muitos deles diagnosticados quando já apresentavam lesão renal. Além disso, o tamanho da amostra analisada foi pequeno, o que pode ter interferido no poder estatístico de detectar diferenças entre os grupos. A desnutrição no indivíduo portador de doença renal crônica é responsável pelo aumento de intercorrências clínicas, elevando o número de infecções e internações ${ }^{(25)}$, o que agrava o quadro nutricional. Além do aumento da morbidade, o aumento da mortalidade em nefropatas desnutridos adultos já está bem documentado na literatura ${ }^{(9,10,26)}$. Em crianças, as consequências da desnutrição são ainda mais importantes, pois seu desenvolvimento cognitivo e motor fica prejudicado, muitas vezes de maneira irrecuperável ${ }^{(5,27)}$. Mesmo quando estáveis do ponto de vista renal e recuperadas em relação ao peso, as crianças não recuperam a estatura ${ }^{(5)}$. Considerando-se os agravos decorrentes da desnutrição e a dificuldade de reverter esse quadro, fica clara a necessidade de intervenção nutricional precoce e efetiva, acompanhada de um controle clínico rigoroso, como importante medida no acompanhamento dessas crianças.

Os avanços das terapias renais substitutivas e do conhecimento da fisiopatologia da doença renal na fase pré-renal possibilitaram melhor acompanhamento e maior sobrevida dos pacientes ${ }^{(28)}$. No contexto atual, o suporte nutricional tornou-se um dos diferenciais no tratamento do portador de doença renal crônica, pois permite melhor qualidade de vida, diminuindo o número de internações e aumentando a disposição geral do paciente.

Com este estudo, concluiu-se que as crianças e adolescentes estudados apresentam alta prevalência de desnutrição, com $44,8 \%$ de baixo peso, $38,8 \%$ de baixa estatura e $22,8 \%$ dos pacientes com IMC para idade abaixo de -2 desvio padrão. Os pacientes apresentaram importantes agravos do estado nutricional, expressos por meio do déficit de peso para idade, estatura para idade e de peso para estatura. Assim, considerando-se os métodos utilizados, não houve diferença do estado nutricional entre as crianças em tratamento conservador de acordo com o clearance de creatinina. 


\section{Referências bibliográficas}

1. Barratt TM, Avner ED, Harmon WE. Pediatric nephrology. $4^{\text {th }}$ ed. Baltimore: Lippincott Willians \& Wilkins; 1999.

2. Brenner BM, Rector FC. The kidney. $6^{\text {th }}$ ed. Philadelphia: Saunders; 2000.

3. Schor N, Srougi M. Nefrologia urologia clínica. São Paulo: Savier; 1998.

4. Mitch WE. Nutrition and the kidney. London: Little Brown; 1993.

5. Walker WA, Watkins JB. Nutrition in pediatrics: basic science and clinical applications. $2^{\text {nd }}$ ed. Hamilton: BC Decker; 1997.

6. National Kidney Foundation Kidney disease. K/DOQI Clinical Practice Guidelines for Chronic Kidney Disease [homepage on the Internet]: Evaluation, classification, and stratification: part 6 - association of level of GFR with complications in adults [cited 2008 Sep 25]. Available at: www.kidney.org/ professionals/KDOQI/guidelines_ckd/.

7. Lowrie EG, Lew NL. Death risk in hemodialysis patients: the predictive value of commonly measured variables and an evaluation of death rate differences between facilities. Am J Kidney Dis 1990;15:458-82.

8. Hakim RM, Levin N. Malnutrition in hemodialysis patients. Am J Kidney Dis 1993;21:125-37.

9. Degoulet $\mathrm{P}$, Legrain $\mathrm{M}$, Reach I, Aimé F, Devriès $\mathrm{C}$, Rojas $\mathrm{P}$ et al. Mortality risk factors in patients treated by chronic hemodialysis. Report of the Diaphane collaborative study. Nephron 1982;31:103-10.

10. Pifer TB, McCullough KP, Port FK, Goodkin DA, Maroni BJ, Held PJ et al. Mortality risk in hemodialysis patients and changes in nutritional indicators: DOPPS. Kidney Int 2002;62:2238-45.

11. Seikaly MG, Salhab N, Gipson D, Yiu V, Stablein D. Stature in children with chronic kidney disease: analysis of NAPRTCS database. Pediatr Nephrol 2006;21:793-9.

12. Pereira AM, Carvalhaes JT. Growth and dietary intake assessment of children with chronic renal failure during predialysis management. J Renal Nutr 1994;4:136-42

13. Kopple JD, Greene T, Chumlea WC, Hollinger D, Maroni BJ, Merrill D et al. Relationship between nutritional status and the glomerular filtration rate: results from the MDRD study. Kidney Int 2000;57:1688-703.

14. Schwartz GJ, Brion LP, Spitzer A. The use of plasma creatinine concentration for estimating glomerular filtration rate in infants, children and adolescents. Pediatr Clin N Am 1987;34:571-90.

15. Fagundes U. Avaliação do estado nutricional e da composição corporal das crianças índias do Alto-Xingu e da etnia lkpeng, através da antropometria, das pregas cutâneas e da impedância bioelétrica [tese de mestrado]. São Paulo: Unifesp; 2003.
16. National Center for Health Statistics [homepage on the Internet]. Growth charts [cited in 2005 Jul 24]. Available from: www.cdc.gov/growcharts/.

17. No authors listed. Use and interpretation of anthropometric indicators of nutritional status. WHO Working Group. Bull World Health Organ 1986;64: 929-41.

18. World Health Organization. Physical status: the use and interpretation of anthropometry. Report of a WHO expert committee. Technical Report Series $n^{\circ}$ 854. Geneva: World Health Organization; 1995.

19. Sociedade Brasileira de Nefrologia [homepage on the Internet]. Censos [cited 2007 Feb 25]. Available from: http//www.sbn.org.br/Censo/2006/CensoSBN2006.ppt

20. van der Heijden BJ, van Dijk PC, Verrier-Jones KJ, Jager KJ, Briggs JD. Renal replacement therapy for end stage renal disease in Europe, 1990-1999. J Am Soc Nephrol. 2002;13:599A

21. Ministério da Saúde. Coordenação-Geral da Política de Alimentação e Nutrição [homepage on the Intenet]. SISVAN [cited 2008 Oct 01]. Avalilable from: http://200.214.130.94/nutricao/sisvan/relatorios/menu_rel_consolidado.php

22. Boot AM, Nauta J, De Jong MC, Groothoff JW, Lilien MR, Van Wijk J et al. Bone mineral density, bone metabolism and body composition of children with chronic renal failure, with and without growth hormone treatment. Clin Endocrinol (Oxf) 1998;49:665-72.

23. Pereira AM, Hamani N, Nogueira PC, Carvalhaes JT. Oral vitamin intake in children receiving long-term dialysis. J Renal Nutr 2000;10:24-9.

24. Norman LJ, Coleman JE, Macdonald IA, Tomsett AM, Watson AR. Nutrition and growth in relation to severity of renal disease in children. Pediatr Nephrol 2000;15:259-65.

25. Herselman M, Moosa MR, Kotze TJ, Kritzinger M, Wuister S, Mostert D. Protein-energy malnutrition as a risk factor for increased morbidity in long-term hemodialysis patients. J Renal Nutr 2000;10:7-15.

26. Qureshi AR, Alvestrand A, Divino-Filho JC, Gutierrez A, Heimbürger O, Lindholm B. Inflammation, malnutrition, and cardiac disease as predictors of mortality in hemodialysis patients. J Am Soc Nephrol 2002;13 (Suppl 1): S28-36.

27. Martin HP. Nutrition: its relationship to children's physical, mental, and emotional development. Am J Clin Nutr 1973;26:766-75.

28. van der Heijden BJ, van Dijk PC, Verrier-Jones KJ, Jager KJ, Briggs JD. Renal replacement therapy in children: data from 12 registries in Europe. Pediatr Nephrol 2004;19:213-21. 